sclerosis, and knee malalignment). Small mean changes over time were noted for these parameters, but all 95\% confidence intervals (CI) overlapped 0. Data from 158 pairs of hip films were analysed prior to and after participation in the long-term safety trial (average duration of treatment, 1 year). Shift table analysis was significant for worsening of hip narrowing $(p=0.029)$ but not other parameters of joint morphology (hip cysts, hip spurs, and hip sclerosis). Mean change analysis demonstrated a change of 0.14 units/year in hip narrowing (95\% CI 0.08-0.20), equivalent to a change of approximately $3 \%$ per year. Mean change analysis demonstrated a change of 0.04 units/year in hip cysts (95\% CI $0.01-0.08$ ), equivalent to a change of approximately $0.2 \%$ per year. These changes were not dose-dependent, and similar changes were noted prior to entry into the long-term safety trial.

Conclusion Celecoxib did not affect index joint x-rays in OA. Modest changes in hip narrowing noted were consistent with the natural history of the disorder.

Sponsored by Pharmacia Corporation and Pfizer, Inc.

\section{SAT0073 PATIENTS WITH KNEE OSTEOARTHRITIS TREATED BY HYLAN G-F 20 VERSUS USUAL TREATMENTS: A MEDICO-ECONOMIC, PROSPECTIVE, RANDOMISED LARGE SCALE TRIAL IN FRANCE, EFFICACY AND SAFETY OUTCOMES}

${ }^{1}$ A Kahan, ${ }^{2} E$ Guemas, ${ }^{2} P L$ Lleu, Synvisc Experts Group ${ }^{3} .{ }^{1}$ University Paris v AP-HP, Cochin Hospital, Paris; ${ }^{2}$ Medical Department, Boehringer-Ingelheim France, Reims, France; ${ }^{3}$ List at the Bottom, Hospitals and Private Offices, France

\subsection{6/annrheumdis-2001.448}

\section{Background}

Objectives To evaluate the efficacy and safety of Hylan G-F 20 over 9 months in the treatment of patients with knee osteoarthritis (OA).

Methods Patients with radiographically proven knee OA, pain on movement ${ }^{3} 40 \mathrm{~mm}$ (VAS) and treated by at least two 10-day NSAIDs courses or by slow-acting anti-OA drugs within the previous 3 or 2 months respectively were enrolled by 81 centres (21 hospitals and 60 private rheumatologists). After stratification on known OA risk factors (patients? age, BMI,...), patients were centrally randomised in Synvisc (S) or in usual treatment (UT) groups. S-patients were treated by 3 intraarticular injections of Hylan GF 20 one week apart and encouraged to stop or spare any other OA treatments. If needed S-patients could be treated by Synvisc in both knees. Efficacy and safety data were collected at baseline, day 28, months 3, 6 and 9 .

Results Out of 518 patients randomised, 506 (253 per group) were treated and evaluable. Demographic data (mean age $=66$ / 66 years, female $=67 / 68 \%$, BMI $=28 / 28 \mathrm{~kg} / \mathrm{m}^{2}$, bilateral knee $\mathrm{OA}=72 / 76 \%$ in $\mathrm{S}$ vs UT groups respectively) were comparable at baseline. Forty-five S-patients (18\%) were treated in both knees.

S-patients showed a better improvement than UT-patients regarding all primary and secondary efficacy outcomes. More especially, mean (SD) changes in S-group versus UT-group were ?3.6 (4.1) vs ?1.6 (4.0) for Lequesne Index, ? 20.1 (19.3) vs ? $8.2(20.5) \mathrm{mm}$ for WOMAC total score and ? 37.4 (22.3) vs ? $24.4(24.0) \mathrm{mm}$ for pain on movement. Therefore additional improvements $(\mathrm{p}=0.0001)$ were observed in S-group for Lequesne Index (+ 18.7\%), WOMAC total $(+24.4 \%)$, and pain on movement $(+21.1 \%)$.
In S-group, global efficacy was assessed as good/satisfactory by $74 \%$ of patients and by $76 \%$ of investigators versus $51 \%$ and $48 \%$ respectively in UT-group, $\mathrm{p}<0.0001$.

Transient local adverse events (pain, swelling) after Synvisc injections were reported in $11.8 \%$ of injections. Gastrointestinal adverse events related to $\mathrm{OA}$ treatment were less frequently reported in S-patients (3.5\%) as compared to UT-patients $(11.9 \%)(\mathrm{p}<0.001)$.

Conclusion This large-scale study confirms the efficacy and safety of viscosupplementation by Synvisc in knee OA over a 9month follow-up.

Acknowledgement Synvisc Experts Group: Prs. Bardin T., Dougados M., Kahan A. (Paris), Mazières B (Toulouse), Trèves R. (Limoges), Vignon E. (Lyon), Drs Auvinet B. (Laval), Naim C. (Marseille), Rossignol O. (Fontenay).

\section{SAT0074 PATIENTS WITH KNEE OSTEOARTHRITIS TREATED BY HYLAN G-F 20 VERSUS USUAL TREATMENTS: A MEDICO-ECONOMIC, PROSPECTIVE, RANDOMISED LARGE SCALE TRIAL IN FRANCE, MEDICO-ECONOMIC OUTCOMES}

${ }^{1}$ A Kahan, ${ }^{2}$ E Guemas, ${ }^{2} \mathrm{PL}$ Lleu, Synvisc Experts Group ${ }^{3} .{ }^{1}$ University Paris v AP-HP, Cochin Hospital, Paris, France; ${ }^{2}$ Medical Department, Boehringer-Ingelheim, Reims, France; ${ }^{3}$ List at the Bottom, Hospitals and Private Offices, France

10.1136/annrheumdis-2001.449

\section{Background}

Objectives To evaluate the medico-economic benefits of Hylan G-F 20 over 9 months in the treatment of patients suffering from knee osteoarthritis (OA).

Methods Patients with radiographically proven knee OA, pain on movement ${ }^{3} 40 \mathrm{~mm}$ (VAS) and treated by at least two 10-day NSAIDs courses or by slow-acting anti-OA drugs within the previous 3 or 2 months respectively were enrolled by 81 centres (21 hospitals and 60 private rheumatologists). After stratification on known OA risk factors (patients? age, BMI,...), patients were centrally randomised in Synvisc (S) or in usual treatment (UT) groups. S-patients were treated by 3 intraarticular injections of Hylan GF 20 one week apart and encouraged to stop or spare any other OA treatments. If needed S-patients could be treated by Synvisc in both knees. Primary outcome was difference in specific knee-OA cost (visits, exams, hospitalisations, treatments and adverse event treatment/prevention related to OA treatments) divided by difference in AUC mean change of Lequesne index between groups. Data were collected at baseline, day 28, months 3,6 and 9 .

Results Out of 518 patients randomised, 506 (253 per group) were treated and evaluable. Both groups were comparable at baseline. Forty-five S-patients (18\%) were treated in both knees. Cost per patient was analysed over 9 months in social security perspective (year 1998 value). All direct and indirect costs were decreased in S-group compared to UT-group (medications ? $40 \%$, non-drug treatments ? $19 \%$, hospitalisation ? $5 \%$, exams ? $2 \%$, seak-leave ? $15 \%$ ) with the only exception of physician visits cost $(+33 \%)$ due to Synvisc injection procedure.

Specific OA total costs (including Synvisc) were 829.12 Euros in S-group and 821.34 Euros in UT-group. Synvisc cost in social security perspective (year 2000 value) is 99.09 Euros per treatment.

AUC mean change in Lequesne Index was 7.9 points in Sgroup vs 9.9 in UT-group ( $p<0.0001$ ). All together the cost/ efficacy ratio (including Synvisc cost) was 7.78 Euros for a 2- 